\title{
Esteatosis en un burro (Equus asinus). Primer reporte en Colombia
}

\section{Steatosis in donkey (Equus asinus). First report in Colombia}

\author{
José Cardona Á, ${ }^{1 *}$ M.Sc, Lázaro Reza G, ${ }^{1}$ Esp. \\ ${ }^{1}$ Universidad de Córdoba. Facultad de Medicina Veterinaria y Zootecnia. Departamento de Ciencias \\ Pecuarias. Montería, Colombia.*Correspondencia: cardonalvarez@hotmail.com.
}

Recibido: Febrero de 2010; Aceptado: Agosto de 2011

\section{RESUMEN}

Se describe un caso de esteatosis en un burro (Equus asinus), castrado, de 15 años de edad, procedente del municipio de San Antero (Córdoba, Colombia), al cual se le detectó ligamento nucal engrosado, duro y doloroso, dando la impresión de un doble cuello y edemas subcutáneos indurados en pared costal, abdominal y pectoral. También presentó masas duras en la unión de músculos semimembranoso y semitendinoso. Por todo lo anterior, mostró dificultad para realizar movimientos coordinados del cuello, nuca y de traslado. Estos hallazgos obedecen principalmente a una deficiencia de selenio y vitamina $\mathrm{E}$, sirviendo como parámetro diagnóstico para la identificación de esta enfermedad en equinos, por lo cual se determinó la actividad eritrocitica de la enzima glutatión peroxidada (GSH-Px), arrojando resultados muy bajos. Este cuadro es también conocido en equinos como enfermedad de la grasa amarilla o esteatitis, que produce degeneración del tejido adiposo, siendo reemplazado por tejido conectivo con depósitos de calcio. Puede estar asociada a miodegeneración nutricional o distrófica (enfermedad del músculo blanco). Es el primer reporte de esta enfermedad en burros (Equus asinus) que se hace en Colombia.

Palabras clave: Burro, deficiencia, esteatosis, glutatión peroxidada, selenio. (Fuente:CAB). 


\section{ABSTRACT}

A case of steatosis in a, 15 years old gelding donkey (Equus asinus), from the town of San Antero (Cordoba, Colombia) is described. the donkey, showed a hard and painful thickened nuchal ligament, giving the impression of a double neck tumors. It was also found an edema in the indurated subcutaneous chest wall, abdomen and chest. It was also found hard lumps in the union of semimembranosus and semitendinosus muscles, which produced difficulty in the movility of the neck and coordinated translation. It is well known that this findings are due to a deficiency of selenium and vitamin $E$, giving them as diagnostic parameters for the presentation of this disease in horses, so we determined the enzyme activity of erythrocyte glutathione peroxidase (GSH-Px), yielding results very low. This condition is also known as yellow fat disease or steatitis in horses and produces degeneration of adipose tissue which is replaced by connective tissue and calcium deposits and may be associated with nutritional or dystrophic miodegeneration (white muscle disease). As far as we know this is the first report of steatosis in donkeys (Equus asinus) in Colombia.

Key words: Deficiency, donkey, glutathione peroxidase, selenium, steatosis. (Source: CAB).

\section{INTRODUCCION}

La esteatosis, también llamada enfermedad de la grasa amarilla (EGA) o esteatitis, es una patología de los equinos en la que se produce degeneración del tejido adiposo, el cual es reemplazado por tejido conectivo con depósitos de calcio (1). La alteración puede estar asociada con miodegeneración nutricional (miopatía nutricional o miodegeneración distrófica), también llamada enfermedad del músculo blanco (2-5), habiéndose descrito la presencia de depósitos de grasa amarilla con necrosis grasa en potros afectados con la enfermedad del músculo blanco (2). En burros se ha reportado el caso en un potrillo con esteatitis y mionecrosis en Nueva Zelanda (6) y en otro de 1.5 meses de edad en Holanda (7). Estos hallazgos obedecen principalmente a una deficiencia de selenio y vitamina $\mathrm{E}(8)$.

El selenio (Se) es un componente de la enzima glutatión peroxidasa (GSH-Px), la cual es una selenoproteína que en las células se ubica en la matriz mitocondrial y en el citosol. Es un agente reductor, cataliza la reducción de peróxidos, superóxidos y radicales hidroxílicos en agua y alcohol, respectivamente $(9,10)$. Al igual que otras selenoproteínas, el sitio activo de GSH$\mathrm{Px}$ contiene selenio ( $\mathrm{Se}$ ) bajo la forma de residuos de selenocisteína incorporada a una cadena polipeptídica conformada por 21 aminoácidos. Se han descrito cuatro isoformas de GSH-Px que difieren tanto en su ubicación como en la especificidad de sustrato. La más importante es la GSHPx celular o clásica, está prácticamente en todas las células, puede reducir el peróxido de hidrógeno e hidroperóxidos orgánicos libres y convertirlos en agua y alcoholes $(10,11)$. La alta correlación existente entre su actividad sanguínea y la concentración plasmática de Se ha permitido emplearla como indicador para evaluar el balance metabólico nutrimental de este mineral en diferentes especies (12-14). La GSH-Px contiene aproximadamente el $75 \%$ del Se sanguíneo, encontrándose en el interior de los eritrocitos a los que se incorpora sólo durante la eritropoyesis (15).

La deficiencia de selenio asociada a la deficiencia de vitamina $E$, permite la acumulación y posterior acción de radicales libre nocivos, destruyendo la membrana celular, proceso al cual las células del músculo esquelético están particularmente expuestas. En situaciones donde ocurre una mayor actividad metabólica, tales como crecimiento, ejercicio, procesos inflamatorios y estrés se produce una mayor demanda tisular de oxígeno, metabolizándose parte de él por la vía 
univalente, generándose una gran cantidad de radicales libres nocivos, $\mathrm{Si}$ la carga de estos supera el sistema de defensa antioxidante, se producen lesiones tisulares al fijarse a los componentes estructurales de la célula, tales como ácidos nucleicos, carbohidratos y lípidos $(1,10,14)$. Los radicales hidroxilicos, peróxidos libres y superóxidos ocasionan lipoperoxidación de la membrana celular al reaccionar con ácidos grasos no saturados, los cuales llevan a la producción de hidroperóxidos $(10,16,17)$, por lo que si los peróxidos producidos no son removidos o inactivados, rompen las membranas celulares y producen la liberación de enzimas lisosomales, las que a su vez producen muerte celular (2). El tejido adiposo afectado se caracteriza por depósitos intersticiales de pigmentos ceroideos originados de la peroxidación lipídica, condición que da el aspecto amarillo anaranjado a la grasa $(1,10)$.

Por lo anteriormente descrito, animales con bajas concentraciones de Se desencadenan una serie de enfermedades, entre las cuales resaltan la esteatosis - enfermedad de la grasa amarilla, así como la miodegeneración nutricional, una enfermedad degenerativa no inflamatoria que afecta la musculatura esquelética y cardiaca de caballos con bajos niveles de Se y/o vitamina $E(1,18,19)$.

Existen diversos reportes de casos clínicos de deficiencia de selenio en caballos, en los cuales se informa que la enfermedad del músculo blanco (EMB) afecta principalmente a potros menores de 2 meses y la esteatosis se reporta en potros desde las 6 semanas hasta los 8 meses de edad $(1,6,20,21)$, Sin embargo, se han descrito casos de esteatosis en caballos mayores de 2 años $(1,3,18,22)$. Los reportes de esteatosis en burros son escasos. Dixon et al (6), informaron un caso de esteatitis y mionecrosis en un burro neonato y Bruijn et al (7), reportaron un caso de EGA en un burro de 1.5 meses de edad. En este último el único signo clínico observado fue edema subcutáneo. No existen datos de la enfermedad en burros adultos, por lo que éste sería el primer reporte de esteatosis en este tipo de animales.
Los signos clínicos se pueden separar en dos síndromes: degeneración miocárdica aguda y miodegeneración esquelética subaguda. Los potrillos con miodegeneración miocárdica pueden ser encontrados muertos, padecer muerte súbita o estar muy débiles. El estrés, especialmente el provocado por el ejercicio, es un factor que precede con frecuencia la aparición de los signos. Se observa un incremento de la frecuencia cardiaca y disnea, pudiendo tener los potrillos grandes dificultades para levantarse y terminan por morir, esto corresponde a la llamada enfermedad del musculo blanco (23).

Las manifestaciones clínicas más representativas de esteatosis descritos en caballos jóvenes y adultos incluyen engrosamiento y endurecimiento del ligamento nucal, siendo ésta la principal manifestación. También, se observan nódulos en la región costal y edema subcutáneo en regiones pectoral e inguinal. De igual forma, se pueden determinar masas más firmes en la unión de músculos semimembranoso y semitendinoso, notándose aumentos de volumen exagerados en esta región. Debido a las nodulaciones y engrosamiento del ligamento nucal, al animal se le hace difícil realizar movimientos de cuello, impidiéndole consumir agua y alimento adecuadamente $(1,8,24)$. Además, se puede observar pelaje hirsuto y quebradizo, rigidez de miembros posteriores y mayor susceptibilidad a infecciones (25).

Araya y Vits (21) y Araya (26), describieron diferentes factores de riesgo que desencadenan la EGA/EMB, dentro de los cuales incluyen dieta deficiente en selenio durante la preñez, exposición a altas cantidades de hierro o azufre en el agua, forraje o suelo (heno contaminado con hongos), suelos ácidos y dietas ricas en grasas no saturadas.

El diagnóstico de la enfermedad se basa principalmente en los antecedentes sobre los factores de riesgo informados, así como los signos clínicos encontrados en el examen físico: Sin embargo, la determinación de glutation peroxiadasa (GSH-Px) eritrocítica se considera concluyente y diagnóstica de esteatosis en equinos $(1,13,21,26)$. 


\section{CASO CLINICO}

Un burro criollo castrado, de 15 años de edad ingresó a las instalaciones de la Facultad de Medicina Veterinaria y Zootecnia de la Universidad de Córdoba, presentando ligamento nucal engrosado, duro y doloroso a la palpación, dando la impresión de un doble cuello (Figura 1), aumentos de volumen y edemas subcutáneos en

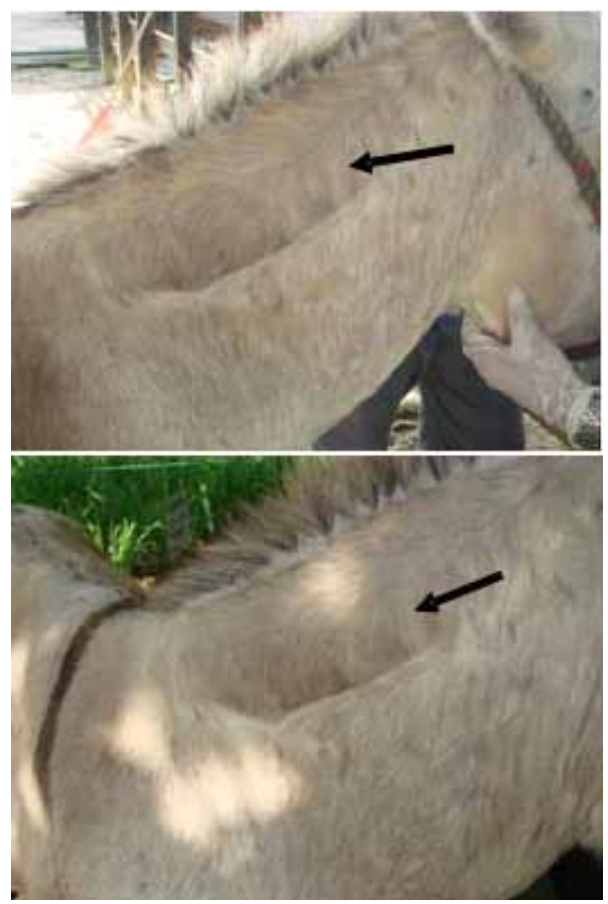

Figura 1. Marcado aumento de volumen de cuello $(\rightarrow)$ y de la cresta nucal en un burro GSHPx deficiente.

pared costal, abdominal y pectorales (Figura 2), masas duras en la unión de músculos semimembranoso y semitendinoso, notándose aumentos de volumen exagerados en esta región (Figura 3). De igual forma, presentó dificultad para realizar movimientos del cuello y nuca y dificultad para realizar movimientos de traslación.

La determinación de GSH-Px en sangre heparinizada del burro indicó una actividad eritrocítica de $28 \mathrm{U} / \mathrm{g} \mathrm{Hb}$ (GSH-Px de referencia en equinos: 130 $-270 \mathrm{U} / \mathrm{g}$ de $\mathrm{Hb}$ ).
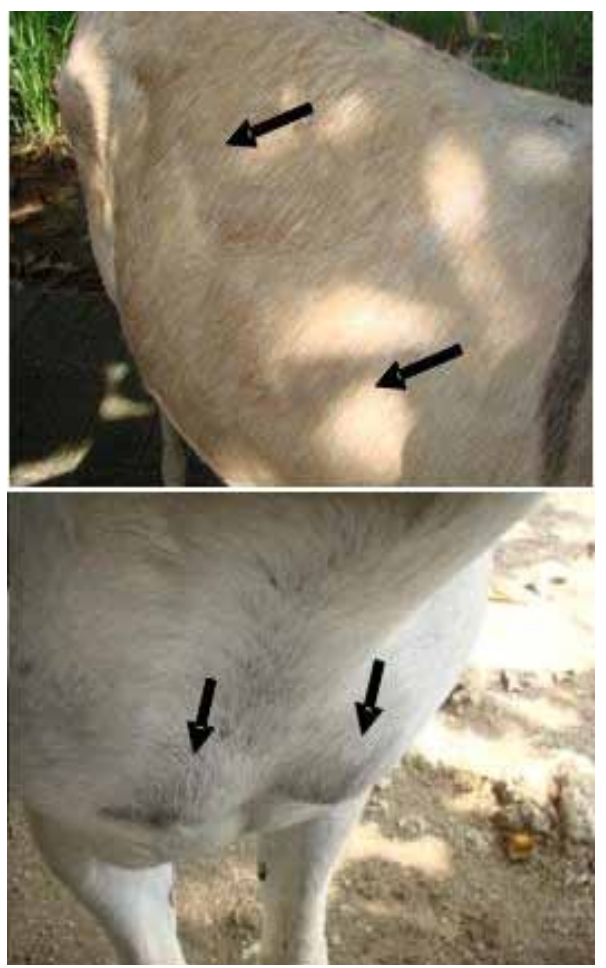

Figura 2. Aumentos de volumen $(\rightarrow)$ y edemas subcutáneos indurados en pared costal, abdominal y pectorales en un burro GSHPx deficiente.

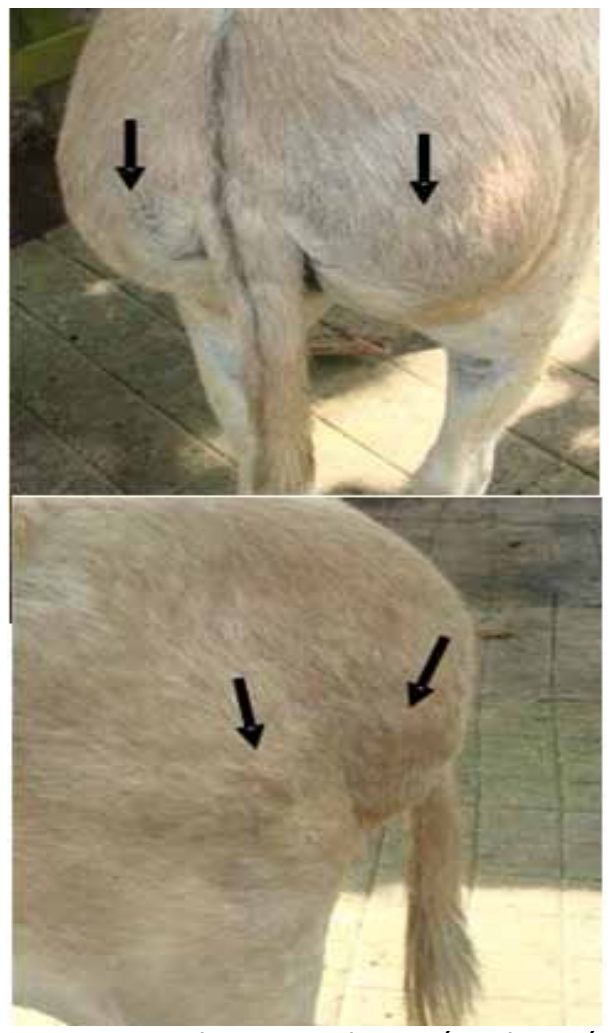

Figura 3. Masas duras en la unión de músculos semimembranoso y semitendinoso de un burro GSH-Px deficiente, notándose aumentos de volumen en esta región $(\rightarrow)$. 


\section{DISCUSIÓN}

El burro adulto macho castrado con signos clínicos compatibles con esteatosis, así como los resultados de la determinación de actividad eritrocítica de glutatión peroxidada (GSH-Px) deficiente, provenía de una región donde se encuentran suelos ricos en azufre (San Antero, Córdoba Colombia), pudiendo ser este el factor de riesgo para la deficiencia de GSH-Px que este animal presentó, de acuerdo a lo indicado por Araya y Vits (21).

Los signos presentados en este burro, engrosamiento del cuello, dolor y rigidez, así como edemas e induraciones en el pecho, tórax y abdomen, concuerdan con las manifestaciones clínicas descritas por Owen et al (22) en caballos adultos, así como Ceballos et al (3) en un caballo de 18 meses y Taylor et al (18) en un pony adulto de 5 años. Sin embargo, Bruijn et al (7), sólo describieron edema en el pecho en un burro de 1.5 meses de edad con enfermedad de la grasa amarilla.

Por otra parte, las manifestaciones clínicas encontradas son compatibles con esteatosis o enfermedad de la grasa amarilla, lo cual fue ratificado por la disminución marcada de la GSH-Px, coincidiendo esto con lo reportado por Freestone y Carlson (8) y Araya (26).
El marcado edema e induraciones con aumentos de volumen subcutáneo duros y circunscritos, ha sido observado en potros y caballos adultos, así como el cuadro caracterizado por engrosamiento de cresta nucal y rigidez de las extremidades y cuello al caminar, como ha sido reportado por Araya et al (1), Bruijn et al (7), Araya y Vits (21) y Araya (26) en caballos con esteatosis.

Los casos reportados en burros por Dixon et al (6) y Bruijn et al (7), han correspondido a neonatos menores de 2 meses, en los cuales no fue determinada la actividad eritrocítica de la GSH-Px, la sospecha diagnóstica se fundó en los hallazgos clínicos, anatomopatológicos e histopatológicos, por lo que no se puede afirmar que la esteatosis reportada fue por deficiencia de selenio.

No se encontraron reportes en la literatura consultada sobre esteatosis en burros adultos en Colombia, lo que se podría atribuir a que sólo algunos casos con niveles bajos de GSH-Px presentan signos clínicos $(1,26)$ o al desconocimiento de la enfermedad, por lo que se hace necesario realizar investigaciones sobre las concentraciones de selenio sanguineo - la actividad eritrocitica de la glutatión peroxidada (GSH-px) en caballos y burros en Colombia.

\section{REFERENCIAS}

1. Araya O, Urzua R, Bustamante H. Efecto del selenato de bario inyectable sobre la actividad de Glutation peroxidasa en caballos a pastoreo Arch Med Vet 2004; 36(1):31-37.

2. Moore R, Kohn C. Nutritional muscular dystrophy in foals. Compend Cont Educ Pract Vet 1991; 13:476-490.

3. Ceballos A, Araya O, Paredes E. Aspectos clínico-patológicos de la esteatosis del equino: descripción de un caso. Arch Med Vet 1996; 28:125-130.
4. Reed S, Bayly W. Equine internal medicine. Philadelphia: WB Saunders Company; 2000.

5. Arias L, Mejía N, Sánchez C, Peláez C, Ceballos A. Actividad de la aspartato aminotransferasa y la creatinkinasa y su relación con la actividad de la glutatión peroxidasa en caballos Pura Sangre Inglés, antes y después de una carrera de 1100 metros. Rev Col Cienc Pec 2004; 17(2):134-140. 
6. Dixon R, Nuttall W, Carthew D. A case of steatitis and myonecrosis in a donkey foal. N Z Vet J 1983; 31(4):62-63.

7. Bruijn C, Veldhuis E, Sloet M. Yellow fat disease in equides. Equine Vet Educ 2006; 18(1):38-44.

8. Freestone J, Carlston G. Muscle disorders in the horse: a retrospective study. Equine Vet J 1991; 23:86-90.

9. Powers S, Lennon S. Analysis of cellular responses to free radicals: focus on exercise and skeletal muscle. Proc Nutr Soc 1999; 58:1025-1033.

10. Chihuailaf $R$, Contreras $P$, Wittwer F. Patogénesis del estrés oxidativo: Consecuencias y evaluación en salud animal. Veterinaria Mexico 2002; 33(3):265-283.

11. Holben $D$, Smith A. The diverse role of selenium within selenoproteins: a review. J Am Diet Assoc 1999; 99:836-843.

12. Rotruck J, Pope A, Ganther H. Selenium: Biochemical role as a component of Glutation peroxidase. Science 1973; 179:588-590.

13. Maylin G, Rubin D, Lein D. Selenium and vitamin $\mathrm{E}$ in horses. Cornell Vet $1980 ; 70: 272-289$.

14. López $A$, Miranda $M$, Hernández J, Castillo C, Benedito J. Glutation peroxidasa (GSH-PX) en las patologías asociadas a deficiencias de selenio en rumiantes. Arch Med Vet 1997; 29:171-180.

15. Oh S, Ganther H, Hoekstra W. Selenium as a component of glutation peroxidase isolated from ovine eritrocites. Biochemistry 1974; 13:1825-1829.
16. McMurray C, Rice D. Vitamin E and Selenium deficiency diseases. Ir Vet J 1982; 36:57-67.

17. Huerta $M$, Ortega $M$, Cobos $M$. Estrés oxidativo y el uso de antioxidantes en animales domésticos. INCI 2005; 30(12): 728-734.

18. Taylor F, Mair T, Brown P. Generalised steatitis in a adult pony mare. Vet Rec $1988 ; 122: 349-351$.

19. Lofstedt J. White muscle disease of foals. Equine Pract 1997; 13:169-183.

20. Kronemann J, Wensvoort P. Muscular dystrophy and yellow fat disease in Shetland pony foals. Neth J Vet Sci $1968 ; 1: 42-49$.

21. Araya O, Vits L. Esteatosis en caballos en el sur de Chile. Div Educ Cont México 1998; 329-332.

22. Owen R, Moore J, Hopkins J, Arthor D. Dystrophic myodegeneration in adult horses. JAVMA 1977; 17:343-349.

23. Colahan P, Mayhew I, Merritt A, Moore J. Medicina y cirugía equina. $4^{a}$ Ed. Editorial. Argentina: Inter-médica; 1998.

24. Contreras $P$, Cubillos V, Araya $O$. Enfermedad del Músculo Blanco en equinos: descripción de dos casos clínicos. Patología Animal 1991; 5:27-31.

25. Rose $R$, Hodgson D. Manual of Equine Practice. 2a Ed. USA: WB Saunders Company; 1999.

26. Araya O. Deficiencia de selenio en caballos. Caballo Chileno 2003; 2:13-16. 